\title{
EFEITO DO PROCESSO HIDROTÉRMICO SOBRE O TEOR DE FIBRA ALIMENTAR EM HORTALIÇAS
}

\author{
GEÍSA FIRMINO TORRES* \\ SILVANA MAGALHÃES SALGADO** \\ ALDA VERÔNICA SOUZA LIVERA*** \\ NONETE BARBOSA GUERRA ${ }^{* * * *}$
}

\begin{abstract}
Considerando a importância das fibras alimentares na redução de doenças crônicas, esta pesquisa teve como objetivo avaliar o efeito do processamento hidrotérmico sobre o teor de fibra alimentar total e suas frações em hortaliças. O material para estudo foi constituído por beterraba (Beta vulgaris rubra L.), cenoura (Daucus carota sativa D.C.) e repolho (Brassica oleracea capiata D.C.). Os teores de fibra alimentar total (FAT), fibra insolúvel (FAI) e fibra solúvel (FAS) foram quantificados pelo método gravimétrico não-enzimático. Os resultados obtidos em g/100 g para amostras cruas de beterraba, cenoura e repolho foram respectivamente, 2,55, 2,62 e 1,84 de FAT; 2,26, 2,65 e 1,80 de FAl e 0,05, 0,00 e 0,00 de FAS. Já as amostras cozidas alcançaram 2,55, 3,17 e 1,96 de FAT, 2,06, 2,63 e 1,66 de FAI, 0,30, 0,42 e 0,19 de FAS para beterraba, cenoura e repolho. Tais resultados permitiram concluir que apesar da fração insolúvel predominar nas hortaliças in natura e cozidas, o processamento hidrotérmico promove aumento da fração solúvel.
\end{abstract}

PALAVRAS-CHAVE: FIBRA ALIMENTAR; HORTALIÇAS.

* Mestranda, Programa de Pós-graduação em Nutrição, Universidade Federal de Pernambuco (UFPE), Recife, PE.

** Doutora em Nutrição, área de concentração Ciência dos Alimentos, Laboratório de Experimentação e Análise de Alimentos (LEAAL),

Departamento de Nutrição, UFPE PE (e-mail: silvanasalgado@ufpe.br).

*** Doutora em Bioquímica Vegetal, LEAAL, UFPE, Recife - PE.

**** Doutora em Ciência dos Alimentos, área de concentração Bromatologia, UFPE, Recife - PE (nguerra@nutrição.ufpe.br). 


\section{INTRODUÇÃO}

Profissionais de saúde têm recomendado maior ingestão de fibras alimentares, incentivando o consumo de frutas e hortaliças como forma de reduzir a incidência de doenças crônico-degenerativas. Já se pode afirmar com razoável segurança os benefícios das fibras alimentares sobre o metabolismo dos lípides e carboídratos, bem como sobre o trato gastrointestinal (LINTAS e CAPPELONI, 1988; SOUSA, SOUZA NETO e MAIA, 2003; LAJOLO e MENEZES, 2006).

Sob o ponto de vista analítico, as fibras alimentares são constituídas por polissacarídeos não-amido (celulose, hemicelulose, gomas e pectinas) e lignina. Entretanto, outros carboidratos (como inulina, amido resistente e $\beta$-glucanas) não são hidrolisados pelas enzimas digestivas, podendo ser considerados também como fibras alimentares (SLAVIN, 2003; JIMÉNEZ-CRUZ et al., 2004; LINTAS e CAPPELONI, 1988).

As propriedades funcionais das fibras são determinadas pela interrelação entre estruturas e características físico-químicas (MONGEAU e BRASSARD, 1989). O conhecimento dessas poderia ser explorado por profissionais da área de saúde e servir como base para recomendações dietéticas mais específicas. Para tanto, tornase necessário identificar as frações solúveis e insolúveis dos alimentos in natura e processados. Segundo a literatura, há fortes indícios de que o processamento hidrotérmico dos alimentos altera os efeitos fisiológicos das frações solúveis e insolúveis.

Esta pesquisa teve como objetivo avaliar o efeito do processamento hidrotérmico sobre o teor de fibra alimentar total e suas frações em hortaliças, usualmente consumidas cruas e cozidas.

\section{MATERIAL E MÉTODOS}

\subsection{MATERIAL}

Constituíram material para estudo beterraba (Beta vulgaris rubra L), cenoura (Daucus carota sativa D.C) e repolho (Brassica oleracea capiata D.C), provenientes de supermercado e da Companhia de Abastecimento e de Armazéns Gerais de Pernambuco, localizados na cidade do Recife. As amostras foram adquiridas aleatoriamente no estádio de maturação em que são rotineiramente comercializadas. 


\subsection{MÉTODOS}

\subsubsection{Tratamento da amostra}

Imediatamente após a sua aquisição, as hortaliças foram transportadas ao Laboratório de Experimentação e Análise de Alimentos da Universidade Federal de Pernambuco. As amostras foram processadas conforme a seqüência operacional ilustrada na Figura 1.

\section{FIGURA 1 - FLUXOGRAMA DE TRATAMENTO DAS AMOSTRAS}

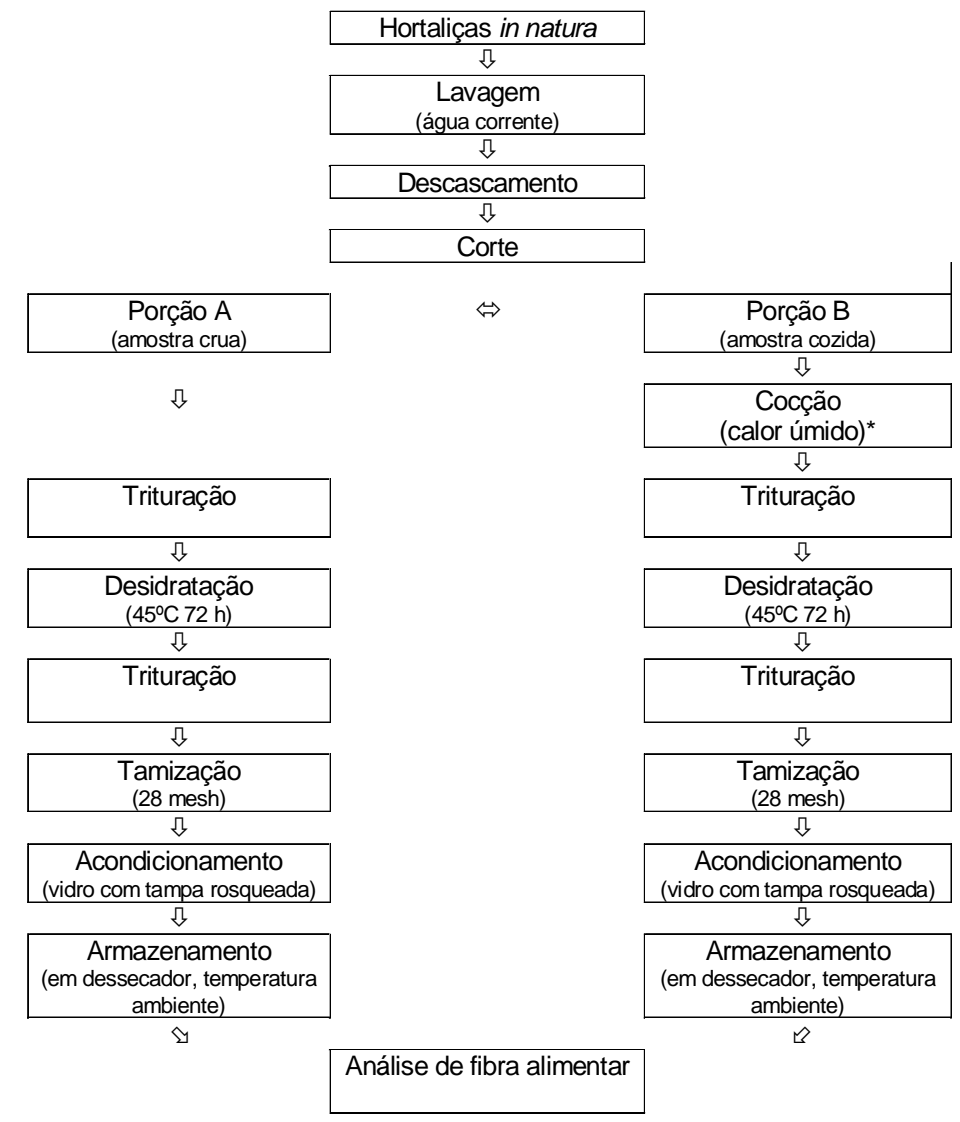

*beterraba $40 \mathrm{~min}$; cenoura $35 \mathrm{~min}$ e repolho $10 \mathrm{~min}$. 


\subsubsection{Determinação da fibra alimentar total e suas frações}

A determinação da fibra alimentar total seguiu o método gravimétrico não-enzimático da AOAC (2002). Para análise das frações insolúveis e solúveis foi utilizado o método gravimétrico não-enzimático daAOAC, adaptado por GUERRA et al. (2004), conforme a Figura 2. Cada lote foi analisado em duplicata perfazendo 10 (dez) resultados para cada hortaliça na forma crua e cozida.

\section{FIGURA 2 - DETERMINAÇÃO DAS FRAÇÕES DA FIBRA ALIMENTAR}

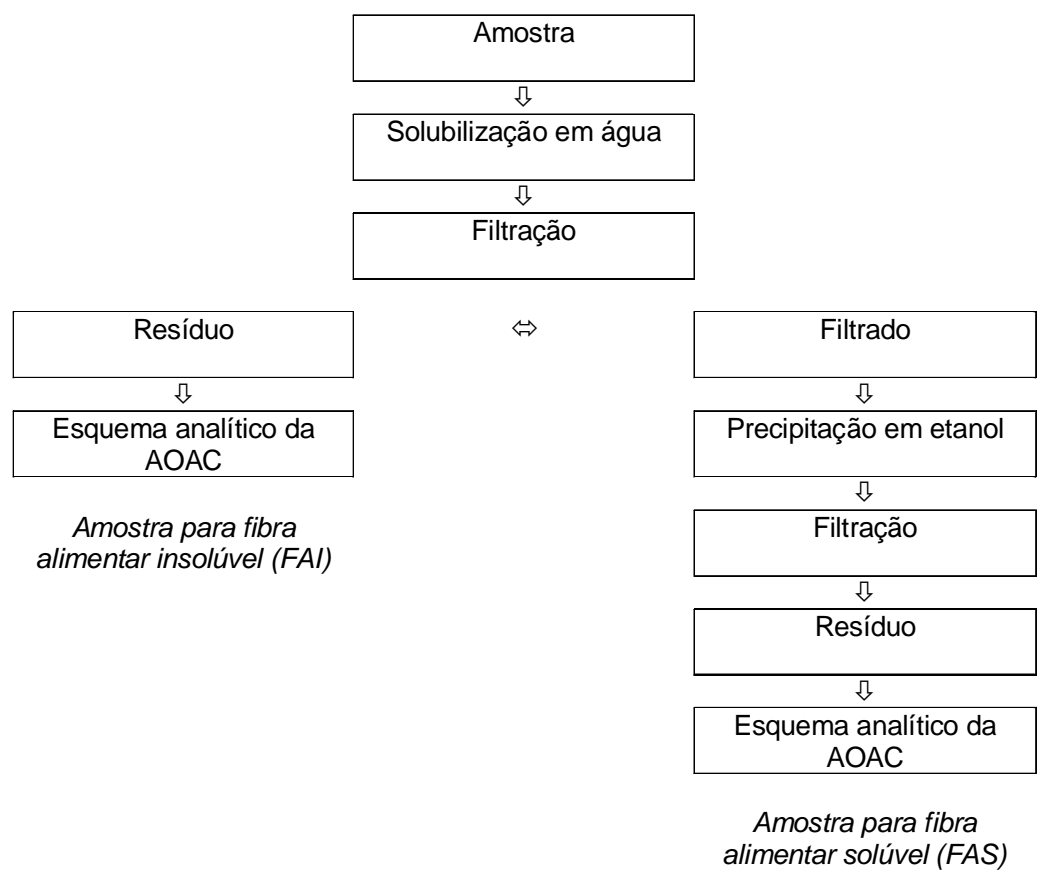

\subsubsection{Análise estatística dos resultados}

O delineamento do experimento seguiu esquema inteiramente ao acaso. Na análise dos resultados aplicou-se o Teste T Pareado, com nível de significância de 5\%, utilizando o software STATSOFT (1996). 


\section{RESULTADOS E DISCUSSÃO}

A análise das Tabelas 1, 2 e 3 demonstra a predominância da FAI, independentemente do tipo de hortaliça e do processo, mesmo com redução de $8,84 \%$ para beterraba, $0,75 \%$ para cenoura e $7,77 \%$ para repolho, após a cocção. Achados semelhantes foram relatados por ZIA-UR-REHMAM, RASHID e SHAH (2004), estudando hortaliças cozidas por diferentes métodos. Verificaram redução da celulose e hemicelulose (mais pronunciada nessa última), a qual foi atribuída à degradação química da celulose à glicose e da hemicelulose à arabinose, xilose e galactose. Tal hipótese já tinha sido levantada por ZIA-UR-REHMAN, ISLAM e SHAH (2003), após a cocção sob pressão e microondas de repolho e cenoura. Trabalhos de SLAVIN (2003); MONGEAU, BRASSARD e VERDIER (1989); GARBELOTTI, MARSIGLIA e TORRES (2003), também relatam que o processamento térmico propicia a solubilização da fibra alimentar em maior ou menor grau conforme o vegetal analisado.

A cocção favoreceu o aumento da FAS, fato já observado por LINTAS e CAPELLONI (1988) e ROSADO, LÓPEZ e HUERTA (1993,) após cocção da beterraba e da cenoura. Em valores absolutos, embora com teores distintos para as frações nas cenouras cozidas, os resultados da FAT aproximaram-se dos determinados neste trabalho. Pesquisa realizada por PHILLIPS e PALMER (1991) evidenciou que cenouras cozidas apresentam geralmente maior teor de fibra solúvel (3-9 g/100 g) em relação à crua. ROSADO, LÓPEZ e HUERTA (1993) constataram $11,2 \%$ de aumento da FAS em amostras de repolho cozidas sem alteração da FAl e FAT, usando método gravimétricoenzimático da AOAC. A literatura relata teor de FAT para repolho cru entre 1,60 a 2,15 g/100 g e para o cozido de 1,3 a 2,4 g/100 g (MONGEAU e BRASSARD, 1989; PLAAMI, KUMPULAINEN e TAHVONEN, 1992).

Com relação à FAT da beterraba, o resultado registrado na Tabela 1 é comparável ao apresentado por GARBELOTTI, MARSIGLIA e TORRES (2003). Convém ressaltar que os teores de FAT das cenouras cruas e cozidas foram similares aos citados por PAUL e SOUTHGATE (1994) $2,9 \mathrm{~g} / 100 \mathrm{~g}$ e $3,1 \mathrm{~g} / 100 \mathrm{~g}$, respectivamente. Esse aumento pode ser 
decorrente da perda de solutos para o meio de cocção (como tanino/ proteína), que contribuiriam para elevação do teor da fibra alimentar como matéria seca.

A discrepância entre os resultados encontrados e a literatura pode estar associada com a maturidade do vegetal, diferença entre cultivares e forma de preparo da hortaliça, mas principalmente com o binômio tempo/temperatura utilizados e/ou procedimento analítico. Para PHILLIPS e PALMER (1991), o tratamento das amostras por liofilização serve como exemplo de tal influência. A etapa de congelamento pode promover expansão na água intracelular, rompendo a integridade da matriz da parede celular que é largamente composta por fibra alimentar, e, conseqüentemente, liberar os constituintes insolúveis para a solução.

TABELA 1- TEORES DE FIBRA ALIMENTAR TOTAL (FAT), FIBRA ALIMENTAR INSOLÚVEL (FAI) E FIBRA ALIMENTAR SOLÚVEL (FAS) EM BETERRABA

\begin{tabular}{c|c|c|c|c|c|c}
\hline \multirow{2}{*}{ AMDSTRA } & \multicolumn{3}{|c|}{ BEIERRABA CRUA } & \multicolumn{3}{c}{ BEIERRABACOZDA } \\
\cline { 2 - 7 } & $\begin{array}{c}\text { FA } \\
(g \%)\end{array}$ & $\begin{array}{c}\text { FAS } \\
(9 \%)\end{array}$ & $\begin{array}{c}\text { FAT } \\
(9 \%)\end{array}$ & $\begin{array}{c}\text { FA } \\
(g \%)\end{array}$ & $\begin{array}{c}\text { FAS } \\
(g \%)\end{array}$ & $\begin{array}{c}\text { FAT } \\
(g \%)\end{array}$ \\
\hline 1 & 2,10 & 0,05 & 2,67 & 1,91 & 0,24 & 2,52 \\
2 & 2,10 & 0,05 & 2,67 & 1,92 & 0,24 & 2,52 \\
3 & 2,49 & 0,07 & 2,48 & 2,31 & 0,39 & 2,73 \\
4 & 2,49 & 0,07 & 2,43 & 2,35 & 0,39 & 2,79 \\
5 & 2,44 & 0,05 & 2,54 & 1,91 & 0,22 & 2,41 \\
6 & 2,42 & 0,04 & 2,54 & 1,91 & 0,22 & 2,38 \\
7 & 2,19 & 0,01 & 2,42 & 2,15 & 0,30 & 2,66 \\
8 & 2,13 & 0,04 & 2,41 & 2,20 & 0,32 & 2,63 \\
9 & 2,10 & 0,06 & 2,65 & 1,93 & 0,36 & 2,46 \\
10 & 2,11 & 0,06 & 2,70 & 1,98 & 0,28 & 2,44 \\
\hline Média & 2,26 & 0,05 & 2,55 & 2,06 & 0,30 & 2,55 \\
Desvio- & 0,178 & 0,018 & 0,114 & 0,178 & 0,070 & 0,141 \\
padrão & & & & & & \\
Coeficiente de & 7,88 & 35,28 & 8,65 & 8,65 & 22,64 & 5,52 \\
variação(\%) & & & & & & \\
\hline
\end{tabular}


TABELA 2 - TEORES DE FIBRA ALIMENTAR TOTAL (FAT), FIBRA ALIMENTAR INSOLÚVEL (FAI) E FIBRA ALIMENTAR SOLÚVEL (FAS) EM CENOURA

\begin{tabular}{c|c|c|c|c|c|c}
\hline \multirow{2}{*}{ AMOSTRA } & \multicolumn{3}{|c|}{ CENOURA CRUA } & \multicolumn{3}{c}{ CENOURA COZIDA } \\
\cline { 2 - 7 } & $\begin{array}{c}\text { FAI } \\
(\mathrm{g} \%)\end{array}$ & $\begin{array}{c}\text { FAS } \\
(\mathrm{g} \%)\end{array}$ & $\begin{array}{c}\text { FAT } \\
(\mathrm{g} \%)\end{array}$ & FAI $(\mathrm{g} \%)$ & $\begin{array}{c}\text { FAS } \\
(\mathrm{g} \%)\end{array}$ & $\begin{array}{c}\text { FAT } \\
(\mathrm{g} \%)\end{array}$ \\
\hline 1 & 2,78 & 0,00 & 2,71 & 2,64 & 0,46 & 3,18 \\
2 & 2,75 & 0,00 & 2,78 & 2,64 & 0,44 & 3,18 \\
3 & 3,07 & 0,00 & 2,92 & 2,87 & 0,53 & 3,46 \\
4 & 3,10 & 0,00 & 2,89 & 2,82 & 0,53 & 3,01 \\
5 & 2,53 & 0,00 & 2,47 & 2,66 & 0,28 & 3,33 \\
6 & 2,53 & 0,00 & 2,51 & 2,64 & 0,32 & 3,36 \\
7 & 2,65 & 0,00 & 2,64 & 2,42 & 0,42 & 2,89 \\
8 & 2,64 & 0,00 & 2,63 & 2,46 & 0,39 & 2,97 \\
9 & 2,23 & 0,00 & 2,34 & 2,54 & 0,44 & 3,15 \\
10 & 2,25 & 0,00 & 2,32 & 2,56 & 0,41 & 3,12 \\
\hline Média & 2,65 & 0,00 & 2,62 & 2,63 & 0,42 & 3,17 \\
Desvio-padrão & 0,293 & 0,000 & 0,211 & 0,142 & 0,080 & 0,180 \\
Coeficiente de & 11,03 & 0,00 & 8,04 & 5,40 & 18,88 & 5,70 \\
variação (\%) & & & & & & \\
\hline
\end{tabular}

TABELA 3 - TEORES DE FIBRA ALIMENTAR TOTAL (FAT), FIBRA ALIMENTAR INSOLÚVEL (FAI) E FIBRA ALIMENTAR SOLÚVEL (FAS) EM REPOLHO

\begin{tabular}{c|c|c|c|c|c|c}
\hline \multirow{2}{*}{ AMOSTRA } & \multicolumn{3}{|c|}{ REPOLHO CRU } & \multicolumn{3}{c}{ REPOLHO COZIDO } \\
\cline { 2 - 7 } & $\begin{array}{c}\text { FAl } \\
(\mathrm{g} \%)\end{array}$ & $\begin{array}{c}\text { FAS } \\
(\mathrm{g} \%)\end{array}$ & FAT $(\mathrm{g} \%)$ & $\begin{array}{c}\text { FAl } \\
(\mathrm{g} \%)\end{array}$ & $\begin{array}{c}\text { FAS } \\
(\mathrm{g} \%)\end{array}$ & $\begin{array}{r}\text { FAT } \\
(\mathrm{g} \%)\end{array}$ \\
\hline 1 & 2,06 & 0,00 & 2,10 & 1,99 & 0,27 & 2,53 \\
2 & 2,04 & 0,00 & 2,09 & 2,01 & 0,24 & 2,55 \\
3 & 1,82 & 0,00 & 1,80 & 1,74 & 0,16 & 1,87 \\
4 & 1,84 & 0,00 & 1,85 & 1,73 & 0,14 & 1,89 \\
5 & 1,64 & 0,00 & 1,69 & 1,30 & 0,13 & 1,41 \\
6 & 1,71 & 0,00 & 1,69 & 1,29 & 0,12 & 1,43 \\
7 & 1,77 & 0,00 & 1,78 & 1,52 & 0,23 & 2,04 \\
8 & 1,82 & 0,00 & 1,79 & 1,53 & 0,25 & 2,00 \\
9 & 1,63 & 0,00 & 1,77 & 1,73 & 0,16 & 1,96 \\
10 & 1,64 & 0,00 & 1,79 & 1,71 & 0,16 & 1,96 \\
\hline Média & 1,80 & 0,00 & 1,84 & 1,66 & 0,19 & 1,96 \\
Desvio-padrão & 0,155 & 0,000 & 0,145 & 0,248 & 0,055 & 0,377 \\
Coeficiente de & 8,63 & 0,00 & 7,92 & 14,98 & 29,79 & 19,18 \\
variação (\%) & & & & & & \\
\hline
\end{tabular}


Nesta pesquisa, o método analítico adotado também exerceu influência nos resultados da FAS em relação a outros autores (NAWIRSKA e KWASNIEWSKA, 2005; LI, ANDREWS e PEHRSSON 2002). A dificuldade em mensurar os componentes solúveis da cenoura e repolho crus deve-se, provavelmente, à heterogeneidade das amostras e redução da precipitação dos constituintes com baixo peso molecular da fração solúvel em etanol a 95\% (GUERRA et al., 2004). Os elevados coeficientes de variação para as hortaliças cozidas podem ser decorrentes da alta viscosidade das amostras que interfere na filtração.

\section{CONCLUSÃO}

De acordo com os resultados apresentados neste trabalho concluise que a fração insolúvel (FAl) é predominante nas hortaliças em ambas as formas analisadas (in natura e cozida). O processamento hidrotérmico favorece o aumento da fração solúvel (FAS) em proporções distintas em função do tipo de hortaliça. A elevada variabilidade para os dados referentes à fração solúvel demonstra a necessidade do emprego de metodologias mais acuradas para sua determinação.

\section{Abstract \\ THE EFFECT OF HYDROTHERMAL PROCESS ON THE CONTENT OF DIETARY FIBER IN VEGETABLES}

Considering the importance of dietary fiber for reducing chronic diseases, the objective of this research was to evaluate the effect of hydrothermal processing on the dietary fiber total content and its fractions in vegetables. The studied material was made up of beetroot (Beta vulgris rubra L.), carrot (Daucus carota sativa D.C.) and cabbage (Brassica oleracea capiata D.C.). The contents of total dietary fiber (FAT), insoluble fiber (FAI), and soluble fiber (FAS) were quantified by the nonenzymatic gravimetric method. The results obtained in $\mathrm{g} / 100 \mathrm{~g}$ for samples of beetroot, carrot and cabbage were respectively 2.55, 2.62, and 1.84 FAT, 2.26, 2.65, and 1.80 FAI, 0.05, 0.00, and 0.00 FAS. For cooked samples the results reached 2.55, 3.17, and 1.96 FAT, 2.06, 2.63 and $1.66 \mathrm{FAI}$, and $0.30,0.42$, and 0.19 FAS for beetroot, carrot and cabbage. These results allowed to conclude that, in spite of the insoluble fraction predominance in cooked and in natura vegetables, the hydrothermal processing brought an increase of the soluble fraction.

KEY-WORDS: DIETARY FIBER; VEGETABLES. 


\section{REFERÊNCIAS}

1 AOAC. Association of Official Analytical Chemist's. Official Methods of AOAC Internacional. 17 $7^{\text {th }}$ ed. Washington, 2002. v.2.

2 GARBELOTTI, M.L.; MARSIGLIA, D.A.P.; TORRES, E.A.F.S. Determination and validation of dietary fiber in food by the enzymatic gravimetric method. Food Chemistry, v.83, p.469-473, 2003.

3 GUERRA, N.B.; DAVID, P.R.S.D.; MELO, D.D., VASCONCELOS, A.B.B.; GUERRA, M.R.M. Modificações do método gravimétrico não-enzimático para determinar fibra alimentar solúvel e insolúvel em frutos. Revista de Nutrição, v. 17, n.1, p.45-52, 2004.

4 JIMÉNEZ-CRUZ, A.; TURNBULL, W.H.; BACARDI-GASCÓN, M.; ROSALES-GARAY, P. A high-fiber, moderate-glycemic index, Mexican stlyle diet improves dyslipidemia in individuals with type 2 diabetes. Nutrition Research, v.24, n.1, p.19-27, 2004.

5 LAJOLO, F.M.; MENEZES, E.W.Carboidratos em alimentos regionales Iberoamericanos. São Paulo: Edusp, 2006. 646 p.

6 LI, B.W.; ANDREWS, K.W.; PEHRSSON, P.R. Individual sugars, soluble and insoluble dietary fiber contents of 70 high consumption foods. Journal of Composition and Analysis, v.15, p. 715-723, 2002.

7 LINTAS, C.; CAPPELONI, M. Dietary fiber content of Italian fruit in raw and cooked vegetables. Food Composition and Analysis, v.42, n.2, p.117-124, 1988.

8 MONGEAU, R.; BRASSARD, R.; VERDIER, P. Measurement of dietary fiber in a total diet study. Journal of Food Composition and Analysis, v.2, p.317-326, 1989.

9 MONGEAU, R.; BRASSARD, R. A comparasion of three methods for analysing dietary fiber in 38 foods. Journal of Food Composition Analysis, v.2, p. 189-199, 1989. 
10 NAWIRSKA, A.; KWASNIEWSKA, M. Dietary fibre fractions from fruit and vegetable processing waste. Food Chemistry, v. 91, p. 221-225, 2005.

11 PAUL, A.A.; SOUTHGATE, D.A.T. Mc Cance and Widdiwsin's: the composition of foods. $5^{\text {th }}$ ed. London: Hermajesty's Stationery Office, 1994.

12 PHILLIPS, K.M.; PALMER, J.K. Effect of freeze-drying and heating during analysis on dietary fiber in cooked and raw carrots. Journal of Agricultural and Food Chemistry, v.39, n.7, p. 29-32, 1991.

13 PLAAMI, S. P.; KUMPULAINEN,J.T.; TAHVONEN, R.L. Total dietary fiber contents in vegetables, fruits, and barries consumed in Finland. Journal Science Food Agricultural, v. 59, p.545$549,1992$.

14 ROSADO, J.L.; LÓPEZ, P.; HUERTA, Z. Dietary fiber in Mexican foods. Journal of Food Composition and Analysis, v.6, p.215222, 1993.

15 STATSOFT, Inc. Statistica for Windows [Computer program manual]. Tulsa, OK, 1996.

16 SLAVIN, J. Impact of the proposed definition of dietary fiber on nutrient data bases. Journal of Food Composition and Analysis, v.16, p.287-291, 2003.

17 SOUSA, P.H.M.; SOUZA NETO, M.A.; MAIA, G.A. Componentes funcionais dos alimentos. Boletim de Ciência e Tecnologia de Alimentos, v.37, n.2, p127-135, 2003.

18 ZIA-UR-REHMAN, M. F.; ISLAM, M.; SHAH, W.H. Effect of microwave and conventional cooking on insoluble dietary fibre components of vegetables. Food Chemistry, v.80, p.237-240, 2003.

19 ZIA-UR-REHINAN, M. F.; RASHID, M.; SHAH, W.H. Insoluble dietary fibre components of food legumes as affected by soaking and cooking processes. Food Chemistry, v.85, p.245-249, 2004. 